\title{
ORIGINAL
}

\section{VELOCIDAD MEDIA DE GANANCIA DE PESO Y ESTATURA EN NIÑOS \\ DE 2 A 10 AÑOS PERTENECIENTES A FAMILIAS DEL ÁREA RURAL DEL MUNICIPIO DE MARINILLA-ANTIOQUIA, COLOMBIA}

Martha Cecilia Álvarez Uribe y Elizabeth Cristina Montoya Puerta

Universidad de Antioquia. Colombia

RESUMEN

Fundamento: El objetivo del estudio fue evaluar la velocidad media de crecimiento de 259 niños de 2 a 10 años, pertenecientes a 150 familias rurales de Marinilla, Colombia.

Métodos: Estudio descriptivo longitudinal prospectivo. En una muestra representativa de familias campesinas productoras de hortalizas y que tuvieran niños de 2 a 10 años, con una confianza del $95 \%$ y un error del $5 \%$ y un sobremuestreo del $20 \%$. Para determinar la velocidad media de crecimiento en peso y estatura se utilizó la metodología propuesta por Baumgartner RN y se comparó con las referencias procedentes del Fels Longitud Study.

Resultados: El 26,8\% de los niños y el 28.9\% de las niñas tuvieron una velocidad media de ganancia de peso $\leq 25$ percentil, en el $60.9 \%$ de los niños y en el $62.8 \%$ de las niñas entre $>25$ y $<75$ percentil y en el $12,3 \%$ de los niños y el $8,2 \%$ de las niñas fue $\geq 75$ percentil. El $44.9 \%$ de los niños y el $33.1 \%$ de las niñas tuvieron una velocidad media de ganancia de estatura $\leq 25$ percentil, en el $44,9 \%$ de los niños y el $52.9 \%$ de las niñas entre $>25$ y $<75$ percentil y en el $10,2 \%$ de los niños y el $14,1 \%$ de las niñas $\geq 75$ percentil.

Conclusiones: La VMC de los niños en estudio no logra compensar el retraso de crecimiento en peso y estatura.

Palabras clave: Niños, velocidad media de crecimiento, estado nutricional.

\section{ABSTRACT}

Average Body Weight and Height Gain Rate in Children from 2 to 10 Years Old, from Families of a Rural Area of the Marinilla Municipality, Antioquia, Colombia

Background: The objective of the present study was to evaluate the average growth rate (AGR) in 2 to 10 years old children ( $\mathrm{n}=$ 259) belonging to rural families from the municipality of Marinilla (Colombia). The results will be taking into account in order to define public health polices aimed to improve the life quality conditions in rural communities.

Methods: In a descriptive longitudinal prospective study a representative sample from rural horticulture families having children aged 2 to 10 years old were selected. The confidence interval, alpha error, and over sample were 95.5 and $20 \%$, respectively. The AGR for weight and high were evaluated according to the method by Baumgarthner, and the results were compared with the Fels Longitudinal Study reference values.

Results: For AGR for weight 26.8 and $28.9 \%$ of boys and girls lassified in the lower 25 percentile, respectively; 60.9 and $62.8 \%$ within the 25 and 75 percentiles, and 12.3 and $8.2 \%$ in the upper percentile of boys and girls, respectively. On the other hand, for AGR for high 44.9 and $33.1 \%$ of boys and girls classified in the lower 25 percentile, 44.9 and $52.9 \%$ within the 25 and 75 percentiles, and 10.2 and $14.1 \%$ in the upper percentile of boys and girls, respectively.

Conclusions: These results showed that the AGR of children in this study could not overcome their delayed weight and high measures.

Key words: Child. Average Growth Rate. Nutritional Status. 


\section{INTRODUCCIÓN}

El municipio de Marinilla está ubicado en la región oriental del departamento de Antioquia, Colombia. Las familias del área rural son productoras de hortalizas a pequeña escala, actividad económica que no genera excedentes monetarios y alimentarios para garantizar la seguridad alimentaria y nutricional de sus integrantes. Las familias no cuentan con agua potable, ni con adecuada disponibilidad de basuras y excretas ${ }^{1}$. En los niños las enfermedades prevalentes son diarrea, parasitosis e infección respiratoria aguda ${ }^{1}$. La cobertura de servicios de salud y de nutrición es baja1. En el estudio realizado por Álvarez y su grupo se encontró que el $54,4 \%$ de las familias estaban en inseguridad alimentaria $^{2}$.

Para establecer los proyectos de desarrollo rural el municipio de Marinilla consideró importante conocer la sostenibilidad de la seguridad alimentaria y nutricional de las familias productoras de hortalizas y entre los aspectos que contempló este estudio fue la evaluación del crecimiento de los niños de 2 a 10 años porque, a pesar de ser un proceso predecible, tiene un comportamiento individual que resulta de la interacción entre los factores genéticos, biológicos y ambienta${ }^{l} \mathrm{~s}^{3} \mathrm{y}$ es un indicador del dinamismo del crecimiento en un período de tiempo en los mismos sujetos a edades específicas ${ }^{4}$.

La disminución de la velocidad de crecimiento corporal es reportada en los países en desarrollo, como consecuencia de la desnutrición calórica proteica, de las enfermedades infecciosas y por las condiciones sanitarias insalubres. Las infecciones ejercen un impacto negativo sobre el crecimiento de los niños y la intensidad de su efecto se presenta por el número de episodios, gravedad, etiología y duración del evento ${ }^{5-7}$. La desnutrición es una de las causas que tiene mayor efecto en el retardo del crecimiento y se encuentra una relación directa entre la severidad de la desnutrición y la deficiencia ponderal ${ }^{8}$.
El objetivo de este estudio fue evaluar la velocidad media de crecimiento en peso y estatura de niños de 2 a 10 años pertenecientes a 150 familias que viven en el área rural del municipio de Marinilla-Antioquia.

\section{SUJETOS Y MÉTODO}

Tipo de estudio: descriptivo longitudinal prospectivo.

Muestra: la unidad de análisis fue la familia, los criterios de inclusión fueron: que vivieran en el área rural del municipio de Marinilla, productoras de hortalizas y estuvieran conformadas con niños de 2 a 10 años. Se calculó una muestra representativa con un $95 \%$ de confianza, un error del $5 \%$ (140 familias) y un sobremuestreo del 20\% (18 familias), previendo la deserción del estudio. La muestra quedo constituida por 168 familias, se retiraron del estudio 18, para una muestra final de 150 . La razón de abandono fue el desplazamiento de la zona por el conflicto armado. Las familias fueron seleccionadas de manera aleatoria del marco muestral, suministrado por la Dirección Local de Salud del municipio de Marinilla.

Los datos antropométricos se tomaron al $100 \%$ de los niños de 2 a 10 años de las familias seleccionadas. En la evaluación inicial se registraron 299 niños, en el primer seguimiento 270 y en el segundo seguimiento 259.

Toma de datos antropométricos: en tres momentos y con un intervalo de seis meses, dos Nutricionistas Dietistas, previa capacitación y estandarización, se tomó en la vivienda del niño el peso en una balanza electrónica con sensibilidad de 50 gramos y se midió la estatura con un tallímetro de 1 milímetro de sensibilidad. La edad del niño se calculó con la fecha de la recolección de los datos y la fecha de nacimiento.

Velocidad media de crecimiento (VMC) para seis meses: con el propósito 
de controlar la permanencia de los niños en el estudio, se tomaron los datos antropométricos en tres momentos: inicial (denominado primer seguimiento) a los seis meses y a los doce meses (denominado segundo seguimiento). Para el cálculo de la VMC se tomaron la variación del peso y la estatura entre el momento inicial y el segundo seguimiento, por considerar que doce meses es un periodo de tiempo apropiado para valorar los cambios en este grupo de edad. Dado que las tablas de referencia para velocidad media de crecimiento del Fels Longitudinal Study, publicadas por Baumgartner ${ }^{9}$, están para seis meses, se acogió la metodología propuesta por este autor de llevar el incremento a seis meses. Así el incremento de peso y estatura en el período (doce meses) se dividió por el número de días entre la evaluación inicial y el segundo seguimiento y se multiplicó por 180 días correspondientes a seis meses ${ }^{9}$. Para clasificar la VMC se establecieron los siguientes rangos: menor del percentil 5, entre el percentil 5 y 25 , mayor del percentil 25 y menor del percentil 75, entre el percentil 75 y 95 y mayor al percentil 95.

Análisis estadístico: por no haber comprobado el supuesto de normalidad de la distribución de las variables antropométricas, el análisis estadístico se realizó con pruebas no paramétricas para muestras pareadas. Se utilizó los programas EPIINFO, SPSS-10.

\section{RESULTADOS}

Descripción de la población: el estudio se realizo en 259 niños, pertenecientes a 150 familias productoras de hortalizas en el municipio de Marinilla-Antioquia. La muestra estuvo formada por los niños que en la primera visita tenían entre 2 y 10 años y que y fueron evaluados en los tres seguimientos, el $53 \%$ de ellos eran de sexo masculino y el $47 \%$ femenino. A partir del primer seguimiento se observa movilidad de los niños entre los grupos de edad y se incluye a los niños que cumplieron 11 años (tabla 1).

Evolución promedio de la ganancia total de peso: las niñas muestran una tendencia al incremento con la edad de la ganancia total de peso $(\mathrm{p}<0,000)$. Las niñas a los 2 años presentaron menor ganancia, entre los 3 a los 6 años el incremento es similar y oscila entre 1,6 y 1,9 kilos, a partir de los 7 años se observa mayor incremento en la ganancia de peso, el cual es consistente hasta los 9 años, a los 10 años obtienen la mayor ganancia (3,7 kilos) (figura 1).

Los niños presentan una tendencia similar a las niñas, salvo a los 3 y 8 años, donde la ganancia promedio decrece, entre los 4 y 6 años la ganancia de peso es muy estable y oscila entre 1,8 y 2,0 kilos, a los 9 años se observa un incremento y la mayor ganancia se encuentra a los 10 años (3,4 kilos) (figura 1). Las niñas a partir de los 7 años presentan mayor ganancia promedio de peso que los niños. Entre los 5 y los 7 años la ganancia fue similar para ambos sexos (figura 1).

Evolución promedio de la ganancia total de estatura: en las niñas la ganancia de estatura decrece con la edad, a partir de los 8 años se observa un incremento de la ganancia de estatura que oscila entre 4,5 y 5,5 centímetros $(p<0,000)$. Los niños presentan la misma tendencia en la ganancia de estatura, aunque se sostiene la ganancia de 5,2 centímetros entre los 4 y 6 años, a partir de los 9 años se incrementa de manera leve la ganancia de estatura $(p<0,000)$ (figura 2$)$. Los niños entre los 2 y 3 años presentan mayor ganancia de estatura que las niñas, entre los 5 y 7 años es similar y a partir de los 7 años las niñas logran mayor ganancia promedio de estatura (figura 3).

Velocidad media de ganancia de peso en gramos y estatura en centímetros en seis meses: en los niños y en las niñas se observa que la velocidad media de ganancia de peso se incrementa con la edad, aunque las niñas 


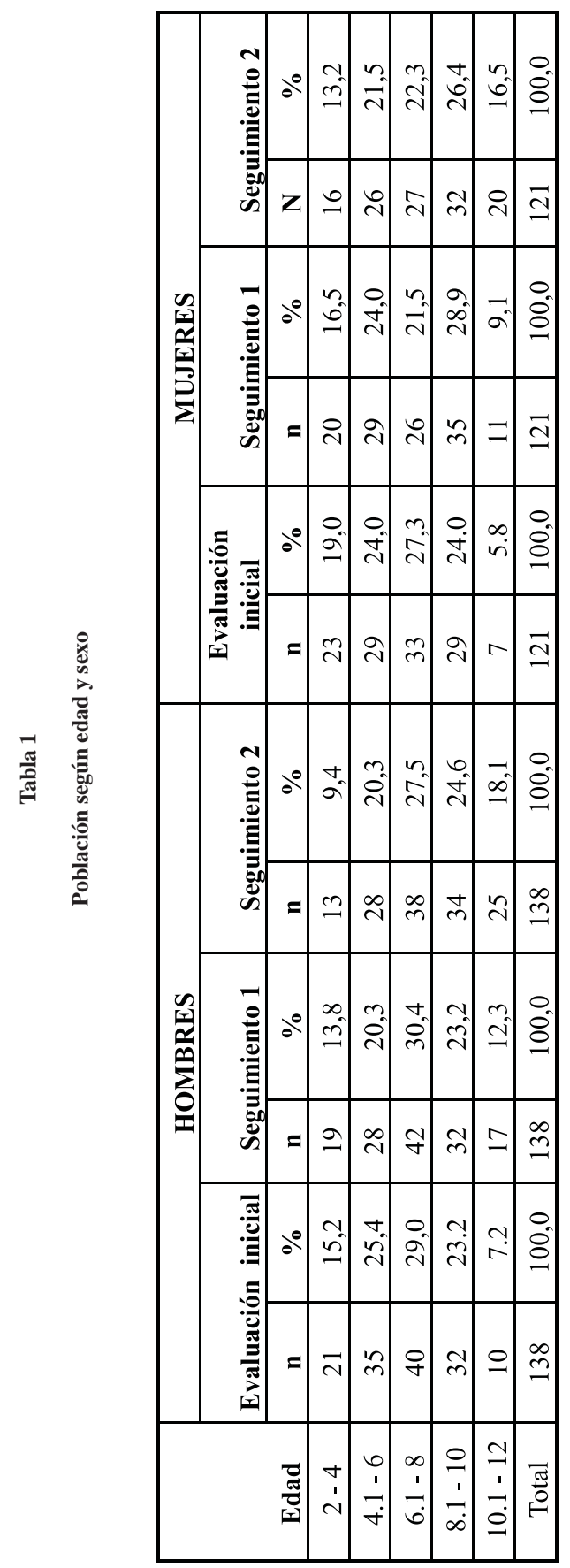


Figura 1

Comparación de la ganancia promedio de peso en kilos con la ganancia promedio de la NCHS
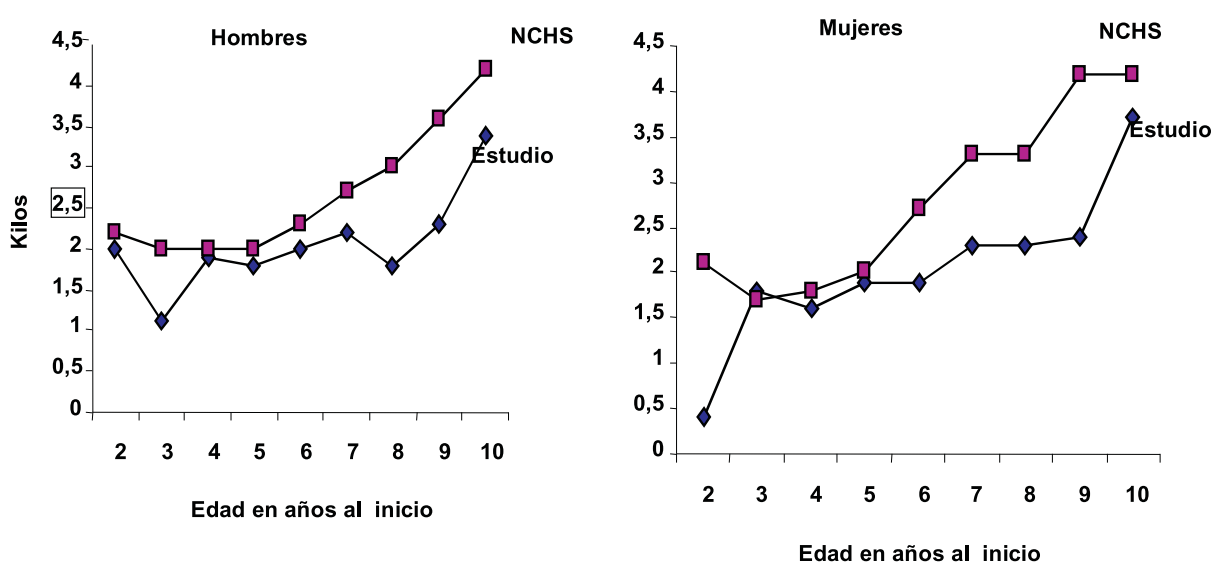

Figura 2

Comparación de la ganancia promedio de estatura en centímetros con la ganancia promedio de la NCHS

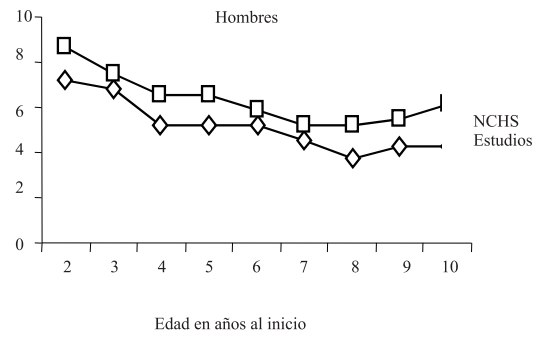

de los dos primeros grupos de edad presentan un incremento menor que los niños y a partir de esta edad superan la velocidad con la cual los niños ganan peso. En éstos la VMC decrece con la edad, pasando de una velocidad promedio de 4 centímetros en el grupo de edad de 2 a 4 años a 2 centímetros en el grupo de 10 a 12 años (tabla 2). En las niñas la disminución en la VMC es menor que en los niños; las de 2 a 4 años presentan la mayor VMC y en las demás se encuentra en promedio una VMC en seis meses de 3 centímetros (tabla 3).

Ubicación percentilar de la velocidad media de ganancia de peso: el $26,8 \%$ de los

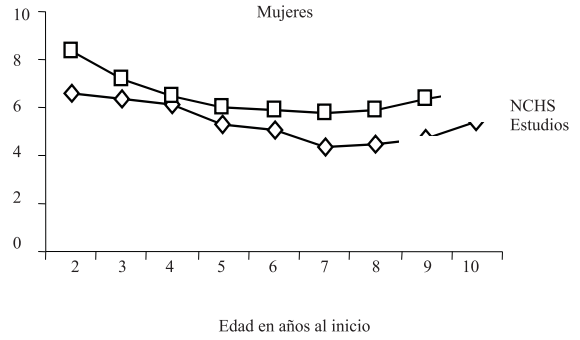

niños y el 28,9\% de las niñas tuvieron una velocidad media de ganancia de peso igual o menor al percentil 25. El 60,9\% de los niños y el $62,8 \%$ de las niñas presentaron una velocidad media de ganancia de peso entre $>25$ y $<75$ percentil y el $12,3 \%$ de los hombres y el $8,2 \%$ de las mujeres tuvieron una velocidad media de ganancia de peso $\geq 75$ percentil (tabla 4).

Ubicación percentilar de la velocidad media de ganancia de estatura: el 44,9\% de los niños y el 33,1\% de las niñas tuvieron una velocidad media de ganancia de estatura que se ubicó en el percentil 25 o por debajo de éste, en el 44,9\% de los hombres y el 
Tabla 2

Velocidad media de ganancia de peso y estatura en seis meses en niños

\begin{tabular}{|l|r|r|r|r|r|r|r|r|r|r|}
\hline \multirow{2}{*}{ Edad } & \multicolumn{9}{|c|}{ Peso en gramos $^{\mathbf{1}}$} & \multicolumn{5}{c|}{ Estatura en centímetros $^{2}$} \\
\cline { 2 - 12 } & $\mathbf{2 - 4}$ & $\mathbf{4 , 1 - 6}$ & $\mathbf{6 , 1 - 8}$ & $\mathbf{8 , 1 - 1 0}$ & $\mathbf{1 0 , 1 - 1 2}$ & $\mathbf{2 - 4}$ & $\mathbf{4 , 1 - 6}$ & $\mathbf{6 , 1 - 8}$ & $\mathbf{8 , 1 - 1 0}$ & $\mathbf{1 0 , 1 - 1 2}$ \\
\hline n & 21 & 35 & 40 & 32 & 10 & 21 & 35 & 40 & 32 & 10 \\
\hline Media & 1058 & 1102 & 1163 & 1173 & 1977 & 4 & 3 & 3 & 2 & 2 \\
\hline Mediana & 976 & 1086 & 1169 & 1187 & 1670 & 4 & 3 & 3 & 2 & 2 \\
\hline d.e $^{3}$ & 722 & 429 & 366 & 497 & 1067 & 1 & 1 & 0,5 & 1 & 0,5 \\
\hline P 25 & 847 & 761 & 880 & 885 & 1013 & 3 & 3 & 2 & 2 & 2 \\
\hline P 50 & 976 & 1086 & 1169 & 1187 & 1670 & 4 & 3 & 3 & 2 & 2 \\
\hline P 75 & 1271 & 1484 & 1423 & 1456 & 2811 & 4 & 3 & 3 & 3 & 3 \\
\hline
\end{tabular}

Tabla 3

Velocidad media de ganancia de peso y estatura en seis meses en niñas

\begin{tabular}{|c|c|c|c|c|c|c|c|c|c|c|}
\hline \multirow[b]{2}{*}{ Edad } & \multicolumn{5}{|c|}{ Peso en gramos ${ }^{1}$} & \multicolumn{5}{|c|}{ Estatura en centímetros ${ }^{2}$} \\
\hline & 2- 4 & 4.1-6 & 6.1-8 & 8.1-10 & 10.1-12 & $2-4$ & 4.1-6 & $6.1-8$ & $8.1-10$ & 10.1-12 \\
\hline & 23 & 29 & 33 & 29 & 7 & 23 & 29 & 33 & 29 & 7 \\
\hline Media $^{2}$ & 888 & 990 & 1238 & 1337 & 2014 & 4 & 3 & 3 & 3 & 3 \\
\hline Mediana & 865 & 1031 & 1138 & 1230 & 1584 & 4 & 3 & 3 & 3 & 3 \\
\hline d.e $e^{3}$ & 451 & 330 & 502 & 708 & 823 & 1 & 1 & 1 & 1 & 1 \\
\hline p 25 & 634 & 728 & 916 & 894 & 1368 & 3 & 3 & 2 & 2 & 3 \\
\hline p 50 & 865 & 1031 & 1138 & 1230 & 1584 & 4 & 3 & 3 & 3 & 3 \\
\hline p 75 & 1075 & 1240 & 1495 & 1560 & 2954 & 4 & 4 & 3 & 3 & 4 \\
\hline
\end{tabular}

$52,9 \%$ de las mujeres la velocidad media se ubicó entre $>25$ y $<75$ percentil y el 10,3\% de los hombres y el $14,1 \%$ de las niñas tuvieron una velocidad media de ganancia de estatura $\geq 75$ percentil (tabla 5 ).

\section{DISCUSIÓN}

Los niños entre los tres y los cuatro años y las niñas entre los dos y los tres años obtuvieron en promedio la menor ganancia de peso, situación que pudo estar afectada por procesos infecciosos a repetición, por ingesta alimentaria baja durante el período evaluado o por una agresión ambiental en el momento de la medición del peso, factores de riesgo que afectan a los niños más peque- ños por su vulnerabilidad nutricional ${ }^{10}$. La ganancia promedio de peso presenta una tendencia ascendente como lo muestra la literatura $^{11}$, sin embargo es inferior a la reportada en las normas de la $\mathrm{NCHS}^{12}$ y se aleja a medida que se incrementa la edad, diferencia que se acentúa en las niñas a partir de los siete años, edad en la cual se inicia la reserva de grasa, denominada rebote de adiposidad, como preparación para el crecimiento y el desarrollo hormonal durante la adolescen$\operatorname{cia}^{13}$. La desnutrición retrasa el inicio de la pubertad e interfiere en la acción de las hormonas del crecimiento y de los factores promotores del crecimiento, de manera especial de la somatomedina $\mathrm{C}^{14}$.

Los niños presentan la misma tendencia de ganancia promedio de estatura reportada 
VELOCIDAD MEDIA DE GANANCIA DE PESO Y ESTATURA EN NIÑOS DE 2 A 10 AÑOS PERTENECIENTES A FAMILIAS..

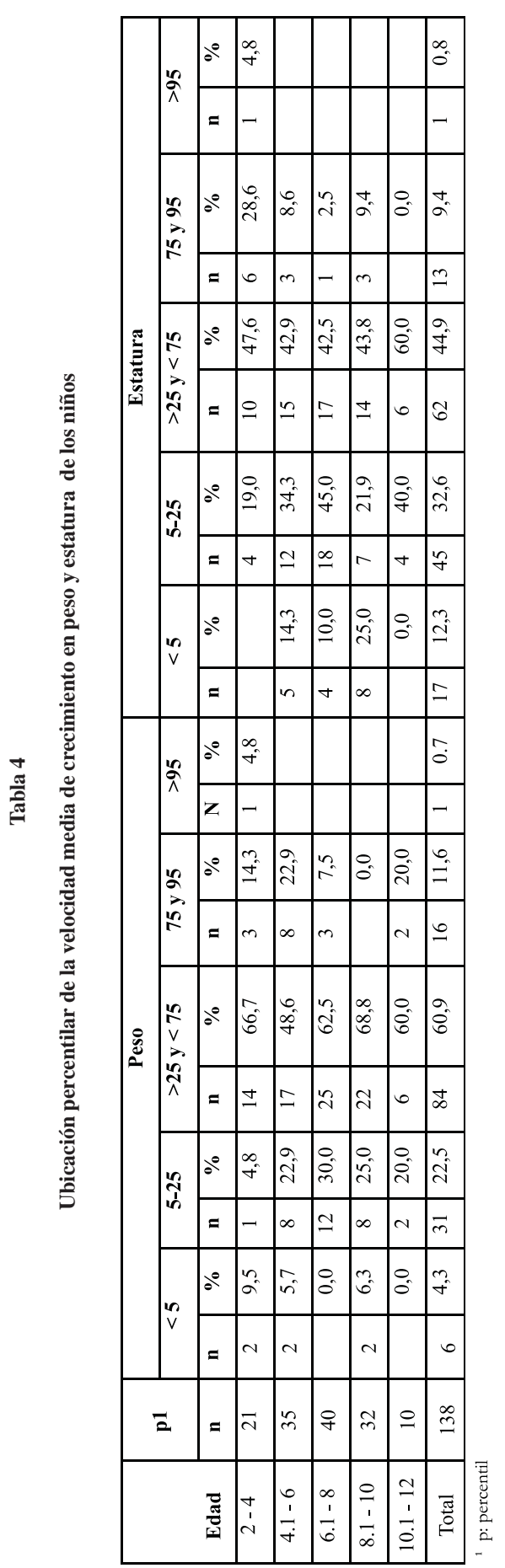

Rev Esp Salud Pública 2004, Vol. 78, N. ${ }^{\circ} 2$ 


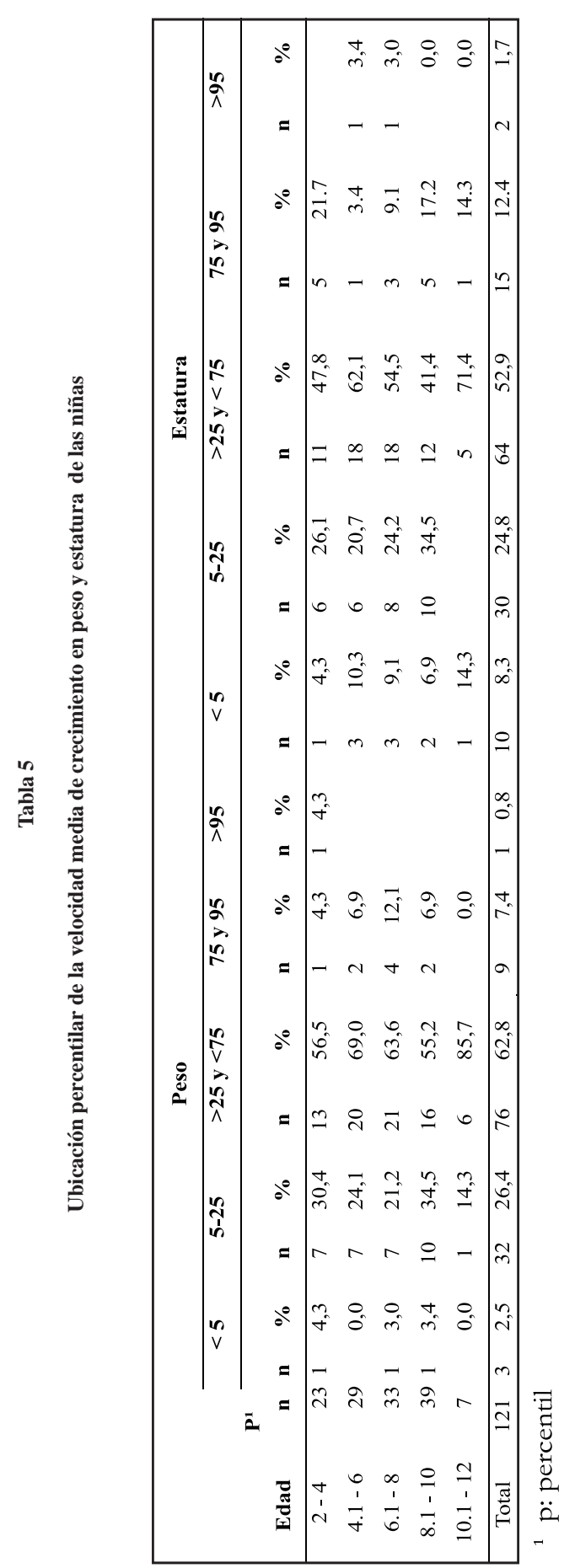


por la literatura ${ }^{11}$. Sin embargo, el incremento promedio obtenido en todas las edades por los hombres y las mujeres fue menor al reportado en las normas de referencia de la $\mathrm{NCHS}^{12}$, situación similar ha sido hallada en niños de América Latina que viven en el área rural $^{15-17}$. Los niños y las niñas del estudio a partir de los 8 años tienden a incrementar la velocidad de crecimiento, pero no lo suficiente para compensar la falla de crecimiento ocurrida durante la infancia, esta misma situación fue documentada en niños de países en desarrollo ${ }^{10,18}$.

Si las condiciones ambientales no favorecen la máxima expresión del crecimiento, los niños no podrán alcanzar su potencial genético y tendrán una estatura baja en su vida adulta ${ }^{19}$. La baja estatura está asociada con alteraciones cognoscitivas ${ }^{20,21}$, menor masa muscular y menor capacidad de trabajo en la vida adulta. Para modificar el retardo del crecimiento se requieren cambios estructurales de los factores que inciden en él y sus efectos son transgeneracionales ${ }^{8,22}$.

El retardo del crecimiento en comunidades pobres es una respuesta biológica a las condiciones adversas que afectan el crecimiento lineal ${ }^{4,16,17,23}$. Martorell en un estudio realizado en niños méxico-americanos, encontró que la pobreza es un predictor significativo de la estatura entre los 2 y los 11 años ${ }^{24}$. Situación en la cual se encuentran las familias de los niños que participaron en el estudio, la actividad económica de sus hogares es la producción a pequeña escala de hortalizas que no genera excedentes monetarios para acceder a la cantidad y calidad de alimentos que necesitan para llevar una vida sana y activa. Tampoco son fuente alimentaria para los integrantes de las familias, porque las verduras no forman parte de su patrón alimentario y además restringen su consumo porque conocen los efectos adversos para la salud de las sustancias químicas que ellos utilizan de manera indiscriminada y en dosis no recomendadas para la salud humana.
Si los niños del estudio continúan con igual velocidad de crecimiento en peso y estatura, no lograrán superar el déficit nutricional y serán adultos en la segunda década del presente siglo, con condiciones diferentes e inferiores a sus pares que tuvieron la oportunidad de crecer y desarrollarse en un medio que les propició mejores condiciones de vida ${ }^{20,21}$.

Le corresponde al gobierno local definir un programa integral de desarrollo rural, con el fin de mejorar sus condiciones de vida e incidir en el crecimiento y desarrollo de los niños del área rural del municipio de Marini$11 \mathrm{a}^{25}$.

\section{BIBLIOGRAFÍA}

1. Municipio de Marinilla. Plan básico de ordenamiento territorial del municipio de Marimilla 1998 -2000: 1-68.

2. Álvarez MC, Restrepo LF. La variedad de alimentos disponibles en el hogar: Metodología para identificar vulnerabilidad a la inseguridad alimentaria y nutricional en hogares campesinos. Rev de Salud Pública y Nutrición 2003;4:1-12.

3. Kain J, Pizarro F. Efecto de un programa de refuerzo alimentario sobre el crecimiento en talla de una población infantil. Arch Lat Nut 1997;47:101- 04.

4. Pinzón P, Laverde J, Franco G. Características del crecimiento y desarrollo en poblaciones de clase media y baja de Bogotá, en el primer año de vida 1985-1986. Bogota: Sociedad Colombiana de Pediatría, 1986:4-25.

5. Stephernson C. Burden of infection on growth failure. J Nutr 1999;129:534-8S.

6. Brown K, RY S, Kanashiro H, OTROS BL. Effects of common illnesses on infant,s energy intake from breast milk and other foods during longitudinal community-based studies in Huascar (Lima), Perú. Am J Clin Nutr 1990:1002-13.

7. Bhan M, Bahl R, Bhandari N. Importancia de los efectos de las infecciones sobre la nutrición infantil y el crecimiento. Nestlé Nutrition Workshop Series Pediatric Program 2000;47:30-31.

8. Saucedo G, Chávez A, Merino B, Madrigal H. Cambios en el déficit de talla de los preescolares en 
la población rural mexicana. Estudios de antropología biológica 2001; X:159-172.

9. Baumgartner RN, Roche AF, Himes JH. Incremental growth tables: supplementary to previously published charts. Am J Clin Nutr 1986;43:711-22.

10. Delgado H. Crecimiento, nutrición e infección en los dos primeros años de vida. Guatemala: Instituto de Nutrición de Centro América y Panamá; 1988.p.1-11.

11. Cusminsky M, Suárez E. Crecimiento y Desarrollo: Salud del niño y calidad de vida del adulto. Organización Panamericana de la Salud publicación científica $\mathrm{N}^{\mathrm{o}}$ 510; 1988; 3-19.

12. Organización Mundial de la Salud. Medición del cambio del estado nutricional. Ginebra: Organización Mundial de la Salud; 1983.p.12-28.

13. Dietz WH. Critical periods in childhood for the development of obesity. Am J Clin Nutr 1994;59:955-9.

14. Restrepo M. Estado nutricional y crecimiento físico. Medellín: Universidad de Antioquia; 2000.

15. Durán M, Ivanovic M, Hazbun J, Ivanovic D. Estado nutricional de escolares rurales de la región metropolitana de Chile. Un estudio comparativo. 1989. Arch Lat Nutr 1996;46:97-106.

16. Sobarzo I, Díaz E, Krause S. Estado nutricional de una población escolar rural chilena. Rev Chil Pediatr 1984;55:109-13.

17. Vargas N, Guardia E, Garrido V. Estado Nutritivo de una población rural chilena(María Pinto). Rev Chil Pediatr 1983;54:282-86.
18. Largo R. Catch -Up Growth during adolescence. Horms Res 1993;39 S:41-48.

19. Tena A, Frinsacho R. Crecimiento antropométrico de la población escolar en las zonas rurales y suburbanas de Durango, México. Arch Lat Nutr 1997;47:105-109.

20. Cravioto J. Malnutrición, desarrollo mental, conducta y aprendizaje. Publicación Científica 1983;450:28-58

21. Toro T, Almagia A, Ivanovic D. Evaluación antropométrica y rendimiento escolar en estudiantes de educación media de Valparaíso, Chile. Arch Lat Nutr 1998;48:201-208.

22. Ramakrishnan U, Martorell R, Schroeder DG, Flores $\mathrm{R}$. Role of intergenerational effects on linear growth. J Nutr 1999;129:544S-549S.

23. Ruel M, Habichit P, Andersen Y, Groohn Y. The mediating effect of maternal nutrition knowledge on the association between maternal schooling and child nutritional status in Lesotho. Am J Epidemiol 1992;135:904-12.

24. Martorell R, Pawson I, Mendosa F, Castillo R. Explicación para la baja estatura en niños méxico americanos. New York: Stanford University; 1987.p.1-10.

25. Amigo P, Bustos P. Programas y políticas referentes al déficit de crecimiento. (Repercusiones de una línea de investigación realizada en Chile). Arch Lat Nutr 1998;48:281-286. 